\title{
Jogo PARA O ENSINO de REABILITAÇÃO NA PESSOA SUBMETIDA A ARTOPLASTIA DA ANCA: ESTUDO DE CASO
}

\author{
JUEGO PARA ENSEÑAR DE REHABILITACIÓN A LA PERSONA SOMETIDA A ARTROPLASTIA DE CADERA: \\ ESTUDIO DE CASO
}

\section{GAME FOR TEACHING REHABILITATION TO THE PERSON UNDERGOING HIP ARTHROPLASTY: CASE STUDY}

DOI 10.33194/rper.2020.v3.s1.3.5765 | Submetido 01/04/2020 | Aprovado 15/10/2020

\author{
Carla Sílvia Fernandes ${ }^{1,2} \mathbb{D}$; Andreia Elisabete Soares ${ }^{3} \mathbb{D}$; Catarina Amaral ${ }^{4} \mathbb{D}$; Eva França Costa ${ }^{5}$; \\ Inês Silva Santos Almeida ${ }^{6}$ (D); Pedro Manuel Ascensão Nogueira7 ${ }^{7}$ \\ 1 - Escola Superior de Enfermagem do Porto; 2 - NursID - Innovation \& Development in Nursing; \\ 3 - Centro Hospital e Universitário São João; 4 - Unidade local de Saúde de Matosinhos; \\ 5 - Centro Hospitalar São João, EPE - Neurocirurgia; 6 - Hospital da Prelada - Unidade de Queimados; \\ 7 - Hospital Lusíadas Porto/ Serviço Atendimento Permanente Adultos/Linha Emergência Interna
}

\section{RESUMO}

Objetivo: 0 estudo teve como objetivo conhecer a opinião dos estudantes de especialização em enfermagem de reabilitação em contexto de ensino clínico sobre a utilização de um Jogo para o ensino dos cuidados de reabilitação à pessoa submetida a Artroplastia da Anca.

Métodos: Trata-se de estudo de caso múltiplo, descritivo e exploratório, no qual foi desenvolvido e aplicado o jogo Reabilit' Arte. Os participantes foram selecionados através de uma amostragem em bola de neve. Para a recolha de dados foi realizada uma breve caracterização sociodemográfica, aplicação do Instrumento de Avaliação de Estratégias de Formação (IAEF), e perguntas abertas sobre as vantagens e desvantagens do jogo. Para a análise das perguntas abertas foi utilizado o software Atlas-ti versão 8.0.

Resultados: A amostra foi constituída por 9 estudantes da pós-licenciatura em enfermagem de reabilitação. Sobre a avaliação da utilização do jogo observa-se que em média da aplicação da escala total os valores variaram entre 80 e 100 muito acima do valor médio da escala. Da análise dos dados, emergiram no âmbito das vantagens da aplicação do jogo as subcategorias: reforço do ensino, facilidade da aprendizagem, participação ativa, motivação e memória visual. No âmbito das desvantagens, com menor frequências são apresentadas o fator tempo e as características dos participantes.

Conclusão: 0 jogo foi avaliado positivamente, revelando que a intervenção do enfermeiro especialista em enfermagem de reabilitação através de um jogo pode ser útil para promover capacidades adaptativas com vista ao autocontrolo e autocuidado nos processos de transição saúde/doença e ou incapacidade.

Palavras-chave: Educação em Saúde; Materiais de Ensino; Artroplastia de Quadril; Enfermagem em Reabilitação

\section{RESUMEN}

Objetivo: El estudio tuvo como objetivo conocer la opinión de estudiantes especializados en enfermería de rehabilitación en el contexto de la enseñanza clínica sobre el uso de un juego para enseñar cuidados de rehabilitación a la persona sometida a artroplastia de cadera.

Métodos: Este es un estudio de caso múltiple, descriptivo y exploratorio, en el que se desarrolló y aplicó el juego Reabilit' Arte. Los participantes fueron seleccionados mediante muestreo de bolas de nieve. Para la recopilación de datos, se realizó una breve caracterización sociodemográfica, la aplicación del Instrumento de evaluación de la estrategia de capacitación (IAEF) y preguntas abiertas sobre las ventajas y desventajas del juego. El software Atlas ti versión 8.0 se utilizó para analizar preguntas abiertas.

Resultados: La muestra consistió en 9 estudiantes de posgrado en enfermería de rehabilitación. Con respecto a la evaluación del uso del juego, se observa que, en promedio, la aplicación de los valores de escala completa varió entre 80 y 100, muy por encima del valor promedio de la escala. Del análisis de datos, las subcategorías surgieron dentro del alcance de las ventajas del juego: refuerzo de la enseñanza, facilidad de aprendizaje, participación activa, motivación y memoria visual. En términos de desventajas, el factor tiempo y las características de los participantes se presentan con menos frecuencia.

Conclusión: El juego fue evaluado positivamente, revelando que la intervención de la enfermera especialista en enfermería de rehabilitación a través de un juego puede ser útil para promover capacidades de adaptación con miras al autocontrol y al autocuidado en los procesos de transición de salud / enfermedad y / o discapacidad. 
Palabras clave: Educación en Salud; Materiales de Enseñanza; Artroplastia de Reemplazo de Cadera; Enfermería en Rehabilitación.

\section{ABSTRACT}

Objective: The study aimed to know the opinion of students specializing in rehabilitation nursing in the context of clinical teaching on the use of a Game to teach rehabilitation care to the person undergoing Hip Arthroplasty.

Methodology: This is a multiple, descriptive and exploratory case study, in which the game Reabilit'Arte was developed and applied. Participants were selected through snowball sampling. For data collection, a brief sociodemographic characterization, application of the Training Strategy Assessment Instrument (IAEF), and open questions about the advantages and disadvantages of the game were carried out. Atlas-ti version 8.0 software was used to analyze open questions.

Results: The sample consisted of 9 postgraduate students in rehabilitation nursing. Regarding the evaluation of the use of the game, it is observed that, on average, the application of the full scale values varied between 80 and 100 , much above the average value of the scale. From the data analysis, the subcategories emerged within the scope of the advantages of the game: reinforcement of teaching, ease of learning, active participation, motivation and visual memory. In terms of disadvantages, the time factor and the characteristics of the participants are presented less frequently.

Conclusion: The game was positively evaluated, revealing that the intervention of the specialist nurse in rehabilitation nursing through a game can be useful to promote adaptive capacities with a view to self-control and self-care in the transition processes of health / disease and or disability.

key words: Health Education; Teaching Materials ;Arthroplasty, Replacement, Hip; Rehabilitation Nursing.

\section{INTRODUÇÃO}

A artroplastia da anca visa restaurar a mobilidade da pessoa, fornecendo um artefacto estável e sem dor na articulação, uma intervenção bem-sucedida depende de um bom planeamento (1) e de uma adequada educação em saúde ${ }^{(2)}$. A intervenção do enfermeiro especialista em enfermagem de reabilitação é crucial de forma a garantir a continuidade de cuidados ${ }^{(3)}$. A artroplastia da anca é uma intervenção cirúrgica, na qual o cliente precisa de 4 a 6 meses para se reabilitar. Contudo, o internamento pode decorrer 4 a 5 dias, o que implica que no momento da alta o cliente ainda apresente um grande nível de dependência. A intervenção do enfermeiro deve ser centrada no cliente e nas suas necessidades e não nas necessidades ou interesses dos profissionais envolvidos. As informações de saúde de qualidade são essenciais para o envolvimento da pessoa, no processo de autogestão e na melhoria da experiência em saúde (2).

0 aumento da expectativa de vida da população aumentou o número de cirurgias e também aumentou os requisitos de reabilitação da pessoa que deseja retornar rapidamente às atividades de trabalho, sociais, desportivas e de lazer ${ }^{(4)}$. Dado o grande e crescente encargo financeiro destes procedimentos, são importantes os modelos de intervenção que demonstrem eficiências, consistindo numa questão de considerável interesse político ${ }^{(5)}$, minimizando as complicações e reinternamentos ${ }^{(6)}$.

As substituições articulares são cirurgias major que requerem uma preparação minuciosa para alcançar um processo tranquilo no cenário peri operatório. Portanto, é necessário que haja um ensino adequado ao longo de todo o processo (7). No nosso ambiente de saúde, cada vez mais complexo, as pessoas requerem informações de alta qualidade para conseguir gerir sua saúde. Embora o acesso à informação tenha aumentado, esta ainda não se traduz numa maior compreensão (2).

Cientes desta necessidade e dado que o enfermeiro especialista em reabilitação deve promover o ensino da pessoa e/ou cuidador com técnicas e tecnologias específicas de autocuidado ${ }^{(3)}$, ousamos em contexto de ensino clinico desafiar a perceção dos estudantes sobre aplicação de jogos como estratégia de educação para a saúde. Os jogos para a saúde estimulam as habilidades de resolução de problemas dos utilizadores, a monitorização e adesão aos comportamentos terapêuticos ${ }^{(8,9)}$. Os jogos educacionais sérios têm demonstrado eficácia na melhoria de vários resultados de saúde ${ }^{(10)}$.

Apesar do recurso à gamificação e aos jogos sérios serem cada vez mais um recurso em saúde, a literatura é escassa quando aplicada à pessoa submetida a artroplastia da anca. Num estudo qualitativo sobre as oportunidades identificadas para a gamificação, os autores salientam, que é necessário abrir possíveis caminhos futuros para explorar o uso de gamificação no percurso da pessoa submetida a artroplastia total da anca e do joelho ${ }^{(11)}$. Os avanços recentes na tecnologia a resultaram numa introdução gradual de intervenções assistidas por computador na reabilitação. Nas últimas décadas, novas tecnologias em reabilitação foram desenvolvidas numa gama de tecnologias empregadas que variam de plataformas de jogos populares e baratos a sistemas altamente especializados com plataformas de hardware e software dedicadas (12).

Apesar da grande evolução das últimas décadas, nomeadamente no que se refere à disparidade de estratégias disponíveis, o ensino ainda vive alicerçado em metodologias tradicionais, nas quais o destinatário é o elemento passivo da aprendizagem (13). Importa salientar que o jogo interliga um conjunto específico de significados, que o jogador se apropria, reconhecendo- 
os, e atuando em função deles. Ou seja, o jogo "fala", o jogador interpreta a "mensagem", dá a sua "resposta" e por sua vez, gera uma nova mensagem. Esta mensagem traz consigo implicações no modo como os indivíduos assimilam, interpretam e concebem os significados ${ }^{(14,15)}$. Isto porque os jogos colocam o sujeito da aprendizagem no centro do processo, como participante ativo (13), no sentido do seu empoderamento.

O conceito de empoderamento da pessoa, com novas informações e novas aprendizagens, permite à pessoa primeiro perceber em termos de autocontrolo, autoeficácia e motivação para gerir um estilo de vida mais saudável. Segundo, capta a pessoa para perceber o ambiente social, as causas relacionadas à saúde e os recursos úteis para a consecução dos objetivos terapêuticos. E por último, descreve as ações que as pessoas levam em consideração para obter um resultado através de comportamentos de adesão terapêutica ${ }^{(9)}$, aspetos esses tão importantes na pessoa submetida a artroplastia da anca.

Neste contexto, o estudo teve como objetivo conhecer a opinião dos estudantes de especialização em enfermagem de reabilitação em contexto de ensino clinico sobre a utilização de um Jogo para o ensino dos cuidados de reabilitação à pessoa submetida a Artroplastia da Anca.

\section{MÉTODOS}

Trata-se de estudo de caso múltiplo, descritivo e exploratório, no qual foi desenvolvido e aplicado um jogo para a pessoa submetida a artroplastia da anca e realizada uma avaliação através da opinião dos estudantes de enfermagem acerca do seu uso.

O jogo foi utilizado no âmbito da pós-licenciatura em enfermagem de reabilitação no ensino clinico da pessoa com afeções orto traumatológicas e conjuntivas, que decorreu entre janeiro e fevereiro de 2020. Para a amostra definiu-se como critérios de inclusão, idade superior ou igual a 18 anos, terem aplicado o jogo em contexto real e participação voluntaria no estudo. Os participantes foram selecionados através de uma amostragem em bola de neve e que voluntariamente aceitaram participar no estudo. A amostragem por bola de neve é indicada quando as amostras com as características do alvo não são facilmente acessíveis, em vez de selecionar uma amostra fixa, cada sujeito que atenda ao critério é selecionado até que o tamanho de amostra necessário seja alcançado ${ }^{(16)}$. Participaram neste estudo 9 estudantes. Uma vez identificados, os sujeitos foram contactados para e esclarecimentos quanto à pesquisa e para o preenchimento e assinatura do consentimento informado, respeitando-se, assim, as especificações da ética em pesquisa. Foi garantida a confidencialidade e anonimato dos dados, com respetiva codificação de todos os dados obtidos.

Para a recolha de dados foi realizada uma breve caracterização sociodemográfica, aplicação do Instrumento de Avaliação de Estratégias de Formação (IAEF) ${ }^{(17)}$ e perguntas abertas sobre as vantagens e desvantagens do jogo na educação para a saúde. Os dados foram analisados com recurso a técnicas descritivas e análise de conteúdo nas perguntas abertas. A interpretação da informação obtida foi efetuada através da análise temática de conteúdo segundo Bardin ${ }^{(18)}$. Foi utilizado o software Atlas-ti versão 8.0 para sistematizar e catalogar o material analisado. As perguntas abertas sofreram uma categorização à priori, identificando elementos em comum em todo o corpus da pesquisa sobre as vantagens e desvantagens do jogo, sujeitos a préanálise, exploração do material, tratamentos dos resultados por frequência e interpretação. Os resultados foram interpretados, estabelecendo-se articulação com a literatura e com o objetivo do estudo.

No que se refere ao Instrumento de Avaliação de Estratégias de Formação (IAEF) é um questionário, de autopreenchimento do qual constam 20 afirmações, com escalas de Likert de 1 a 5 (1 - Discordo fortemente 2 - Discordo; 3 - Indiferente; 4 Concordo; 5 - Concordo Fortemente). A mesma incorpora três dimensões: avaliação da satisfação, avaliação sobre os resultados e avaliação sobre o procedimento, apresentando uma elevada consistência interna com um alfa de Cronbach de 0,952 . O seu preenchimento pode obter uma classificação de 20 a 100 . Na aplicação dos questionários, apenas foram integrados os estudantes que, voluntariamente integraram o estudo, em conformidade com a informação do seu consentimento.

\section{0 jogo}

O jogo intitula-se Reabilit'Arte (figura 1) e inclui um conjunto de cartas e materiais para exemplificar alguns autocuidados, designadamente boneco, canadianas, simulador carro, banheira, cadeira, banco e escadas.

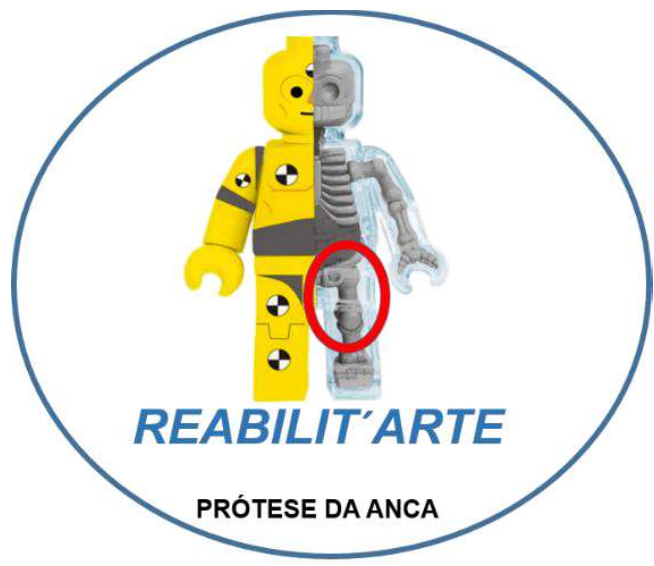

Figura 1: Logotipo jogo

O Reabilit 'Arte é um jogo de cartas em que o progresso é determinado pela capacidade de resposta do jogador às questões sobre conteúdos dos cuidados referentes à artoplastia da anca. O jogo é uma forma lúdica de aprender e sistematizar a informação, muito importante para a reabilitação após a cirurgia de colocação de prótese da anca. Os dados das cartas incidem sobre o autocuidado transferir, andar com auxiliar de marcha, sentar, subir e descer escadas, entrar e sair da viatura e da banheira, exercícios de 
fortalecimento muscular e adaptação do edifício residencial (figura 2 ).

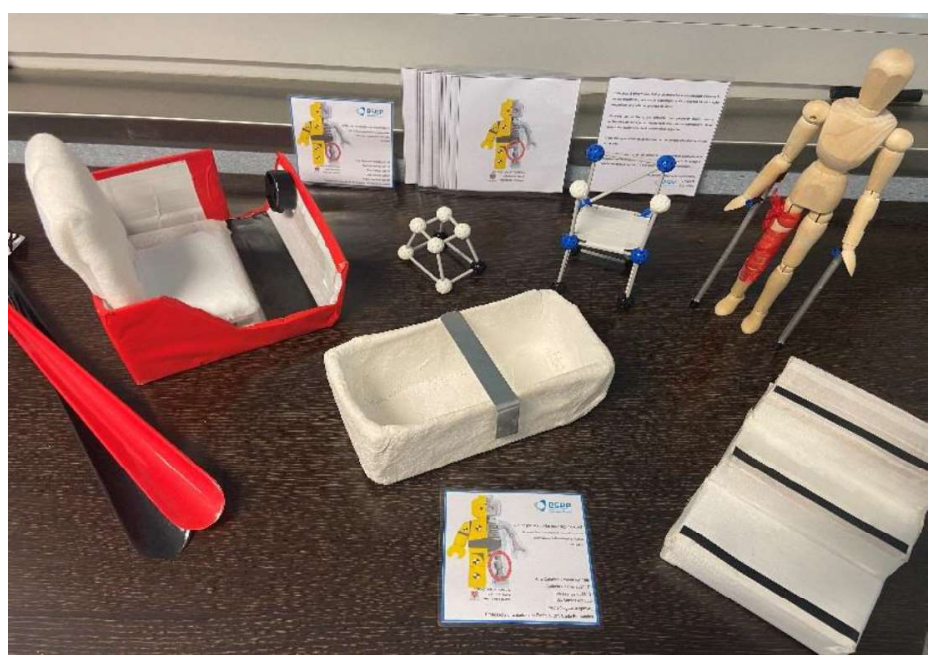

Figura 2: Elementos do jogo

O utente escolhe um cartão e, em seguida, individualmente ou num pequeno grupo, com o enfermeiro de serviço ou mesmo com uma pessoa significativa, deve responder assertivamente à problemática sugerida (figura 3: exemplo de cartão). Cada item que referir sobre os autocuidados devem ser demonstrados com o boneco articulado e equivalem a 1 ponto. No final o utente deve ser capaz de observar a sua própria evolução e o somatório de mais pontos quererá dizer que está mais perto de atingir o objetivo de toda a equipa, ou seja, a sua autonomia para a reabilitação.
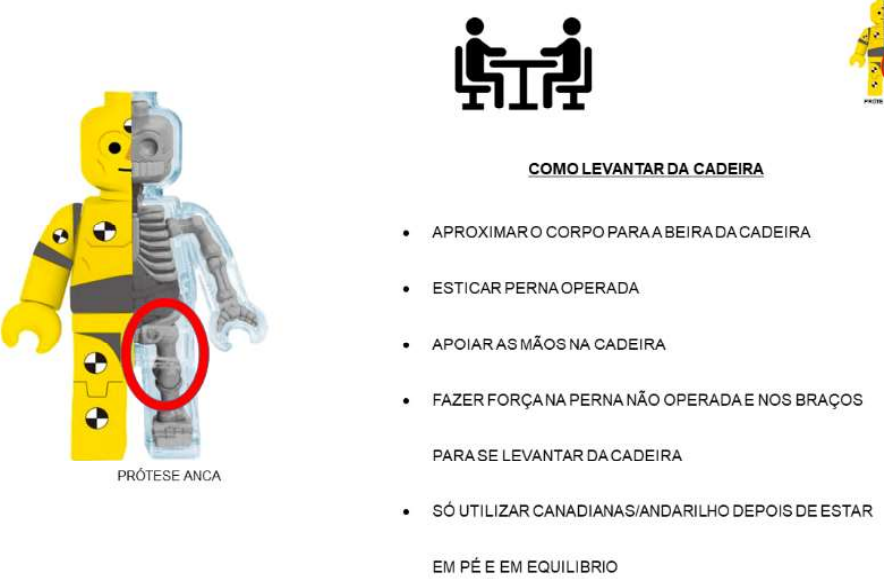

Figura 3: Exemplo de cartão

\section{RESULTADOS}

A amostra foi constituída por 9 estudantes com uma média de idades de 35,1 anos, sendo 8 do género feminino. No que respeita à experiência profissional, trabalham em média há 12,8 anos, numa experiência que varia entre os 5 e os 17 anos. 0 gráfico que se segue apresenta a opinião dos enfermeiros sobre a utilização do jogo, através da aplicação do instrumento IAE. O gráfico apresenta os valores da escala total (amplitude da subescala: 20-100) e das suas diferentes subescalas, avaliação da satisfação (amplitude da subescala: 8-40), avaliação sobre os resultados (amplitude da subescala: 6-30) e avaliação sobre o procedimento (amplitude da subescala: 6-30).

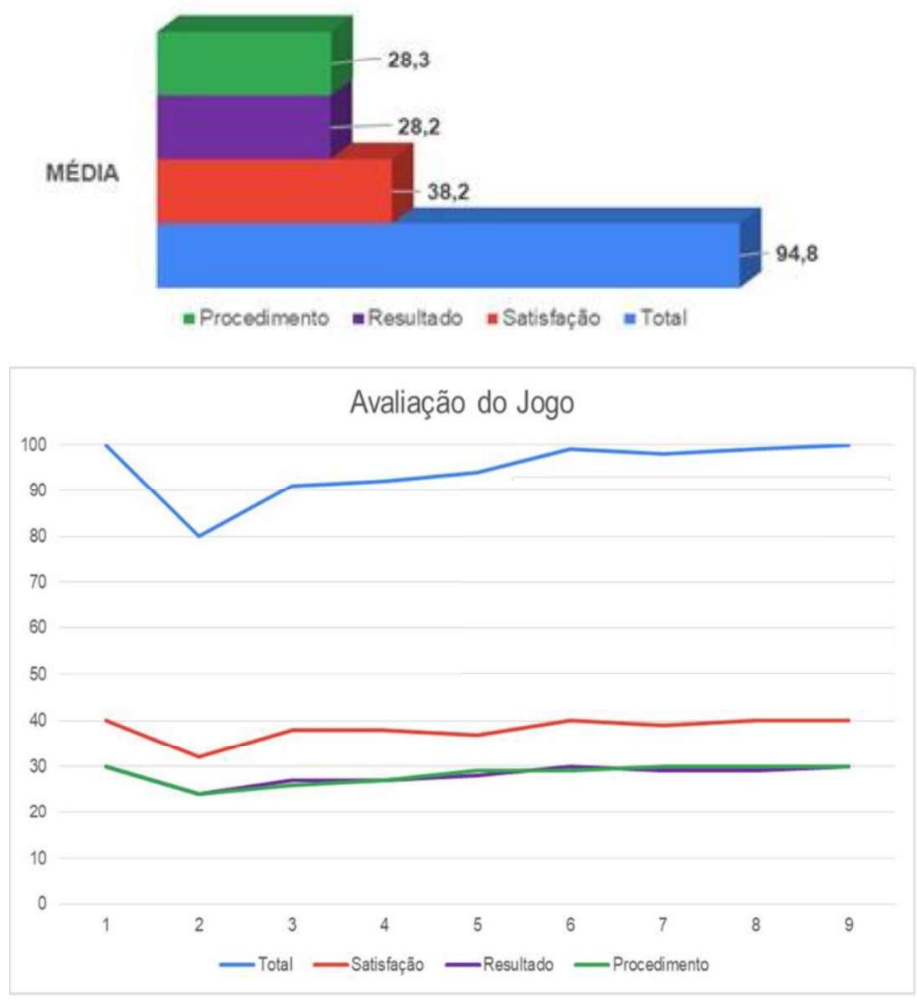

Gráfico 2: Avaliação do jogo

Sobre a avaliação da utilização do jogo observa-se que em média da aplicação da escala total os valores variaram entre 80 e 100 muito acima do valor médio da escala (ponto médio 60), com uma média de 94,8. No que se refere à dimensão avaliação da satisfação os valores oscilaram entre 32 e 40 (ponto médio 24),com uma média de 38,2. A dimensão avaliação sobre os resultados obteve uma amplitude entre 24 e 30 (ponto médio 18),com uma média de 28,2. A dimensão avaliação sobre o procedimento variou entre 24 e 30 (ponto médio 18), com uma média de 28,3.

Os dados que emergiram da análise das perguntas abertas foram organizados por categorias e subcategorias, e são apresentados na Figura 4.

Da análise dos dados, emergiram no âmbito das vantagens da aplicação do jogo as subcategorias: reforço do ensino, facilidade da aprendizagem, participação ativa, motivação e memória visual. No âmbito das desvantagens, com menor frequência são apresentadas o fator tempo e as características dos participantes. Cada uma destas áreas é explorada de seguida na discussão. 


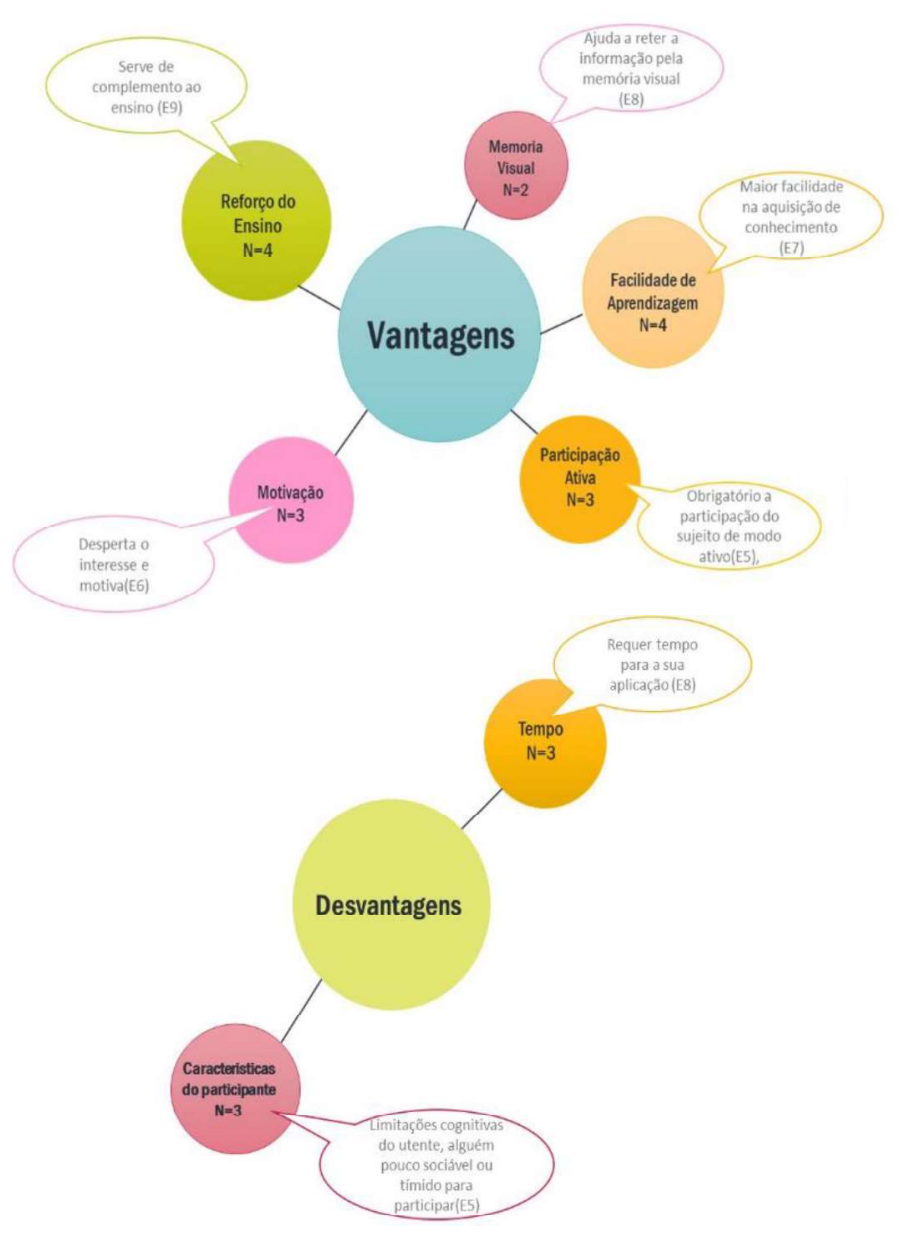

Figura 4: Temas emergentes

\section{DISCUSSÃO}

Os jogos sérios são cada vez mais recomendados como técnicas eficazes para melhorar a educação em saúde ${ }^{(10)}$, recorde-se que o nosso estudo pretendia conhecer a opinião dos estudantes de especialização em enfermagem de reabilitação, em contexto de ensino clínico, sobre a utilização de um Jogo para o ensino dos cuidados de reabilitação à pessoa submetida a artroplastia da Anca.

A utilização do jogo Reabilit 'Arte como estratégia de ensino permitiria ao estudante ir ao encontro de competências especificas do enfermeiro de reabilitação, designadamente conceber e implementar planos de intervenção com o propósito de promover capacidades adaptativas com vista ao autocontrolo e autocuidado nos processos de transição saúde/doença e ou incapacidade ${ }^{(3)}$, neste caso na pessoa submetida a uma artroplastia. Dada a crescente prevalência desta cirurgia, juntamente com a redução do tempo de permanência, os utentes precisam de todo o tipo de informação para uma tomada de decisão consciente (2).

Atualmente é enfatizado o papel ativo das pessoas na melhoria de sua condição de saúde. Assim, a educação e as intervenções de promoção da saúde devem ser repensadas como processos que podem aumentar o controlo e melhorar as condições de vida das pessoas (9). São diversos os estudos desenvolvidos com jogos educacionais sérios em diferentes áreas da saúde, que vão desde à abordagem preventiva até a gestão de doenças crônicas, nos mais diversos formatos, com um pico de concentração nos últimos anos ${ }^{(10)}$. Na pesquisa realizada, a este nível, não foram identificados jogos nesta população alvo, ou seja, nos utentes submetidos a artroplastia da anca, e os estudos desenvolvidos incidem essencialmente sobre o ensino pré-operatório $(4,5,6)$.

No que se refere aos jogos, conforme nos referem estes autores, o manifesto "lúdico" delimita o jogo como um fenômeno ancestral, contemporâneo à própria humanidade, cuja relevância atingiu um novo patamar mediante a ascensão das tecnologias digitais ${ }^{(14)}$. Os jogos educacionais visam principalmente aumentar a consciencialização e conhecimento entre os jogadores. No entanto, o objetivo final dos jogos educativos de saúde devem incluir uma mudança de comportamento nos utilizadores, produzindo assim um efeito duradouro (10). Embora os jogos tenham sempre feito parte da história da humanidade, a sua utilização como estratégia de ensino de educação para a saúde tem ganhado força nos últimos anos.

Sobre a utilização do jogo Reabilit 'Arte, da análise dos dados recolhidos verifica-se que a maioria dos participantes incluídos no estudo concorda com a utilização do jogo tendo sido obtido valores elevados com a aplicação do instrumento IAEF, tanto no valor da escala como nas várias dimensões do instrumento. 0 valor mais baixo, embora ainda elevado é obtido na dimensão avaliação do resultado. Estes resultados referem-se às aquisições (os recursos adquiridos pelo jogo), os efeitos (a utilização dos recursos em situação), e as imagens (as representações que foram construídas sobre as aquisições e efeitos) (17). Estes resultados corroboram, os dados deste estudo onde os autores referem que os enfermeiros têm uma maior propensão para atividades cinestésicas, preferindo uma abordagem prática e ativa de educação, realçando que as pesquisas mostram que as estratégias de aprendizado ativo podem aumentar a aprendizagem e satisfação ${ }^{(19)}$.

Embora os resultados demonstrem que os jogos sérios em termos de eficácia para tais propósitos são promissores, sua implementação nas modalidades de prevenção, tratamento ou treino em saúde é dificultada pela falta de compreensão dos conceitos subjacentes entre profissionais de saúde ou até desconfiança (20). Apesar de os discursos dos participantes enalteçam os aspetos positivos com a utilização dos jogos aquilo que se observa, são o recurso a estratégias tradicionais, em que o sujeito assume um papel passivo. Destaca-se a necessidade de mudança da educação em saúde de um paradigma de prescrição - no qual os utentes seguem a saúde e recomendações de tratamento dos profissionais - para a educação baseada no empoderamento -através do qual os utentes podem aprender a ser responsavelmente ativos no gerenciamento de sua própria saúde ${ }^{(10)}$. Segundo este autor, também a este nível é importante uma prática baseada em evidências, resumindo-a na seguinte definição: a prática baseada em evidências ajuda os 
enfermeiros a oferecer assistência ao utente de alta qualidade, com base em pesquisas e conhecimentos, e não porque 'é assim que sempre fazemos', ou com base em tradições, mitos, palpites, conselhos de colegas ou livros didáticos desatualizados ${ }^{(19)}$.

Sobre a análise das vantagens dos jogos referidas pelos participantes sobressaem aspetos relacionados com a motivação, participação ativa do sujeito, reforço do ensino, facilidade na aprendizagem e memória visual. No que se refere à motivação, num estudo de revisão sobre os resultados da utilização de jogos, os autores salientam as componentes do processamento motivacional, de atenção, relevância e confiança, como preditores significativos de satisfação com a utilização de jogos (21). Um outro estudo de revisão salienta o maior envolvimento do utilizador, parcialmente explicada pela aprendizagem ativa nas tarefas executadas pelos utilizadores ao interagir com um jogo educativo (4), aspeto esse salientado pelos participantes na aprendizagem ativa e como facilitador da aprendizagem.

Os participantes salientaram a importância da utilização do jogo como reforço a outras estratégias de ensino. Embora o jogo congregue todos os cuidados referentes à artroplastia da anca, não poderá existir por si só, mas como complemento a outras abordagens de educação para a saúde, no seu caracter multidimensional, essencial para o empoderamento da pessoa. Este aspeto é reforçado num estudo qualitativo sobre as necessidades da pessoa submetida a artroplastia da anca e do joelho em que salientam a importância da educação multimodal adaptada às necessidades individuais, preferências e experiências, integrando estratégias de educação em diferentes formatos e em diferentes momentos ${ }^{(2)}$.

No que se refere às desvantagens do jogo, embora em menor frequência sobressai os aspetos relacionados com as características dos participantes, nomeadamente limitações cognitivas, timidez, menor interesse por estratégias inovadoras. Com efeito, ainda é difícil avaliar a relação entre jogos sérios e modelos educacionais capazes de capacitar os processos de aprendizagem dos utentes como atores. Além disso, um aspeto crítico que precisa ser considerado nesse contexto é se há diferenças nos utilizadores quanto à sua atitude e aceitação em relação à aprendizagem baseada em jogos ${ }^{(10)}$. Embora existam diferentes estilos de aprendizagem, a maioria dos utilizadores são cinestésicos, além de que os jogos podem beneficiar um maior número de utilizadores muitos por congregar diferentes estímulos ${ }^{(16)}$. O foco deverá ser promover no utilizador o desenvolvimento de competências, alicerçadas num processo de construção de saberes refletido com a implicação do próprio sujeito ${ }^{(13)}$.

No que se refere ao constrangimento de tempo, este está relacionado não apenas com o tempo necessário ao desenvolvimento do jogo, mas também relacionado com o período necessário à sua implementação. Este aspeto é referido por outros autores, em que apontam a restrição de tempo como desencorajador à utilização de jogos ${ }^{(13,19)}$.
Conforme referido no início deste artigo existe ainda uma escassez da utilização de jogos no ensino à pessoa submetida a artroplastia da anca, o que não nos permite comparar os resultados nesta discussão ${ }^{(11,12)}$. Observam-se porém alguns dados mas referente à utilização dos "exergames" designadamente com recurso à Nintendo e WI para a reabilitação ${ }^{(22-24)}$.

Perante estes dados podemos afirmar que os jogos sérios para a saúde combinam profundamente as estratégias cognitivas e os processos motivacionais de empoderamento do utente. Os jogos podem ser úteis para aumentar a capacidade dos utentes de tomarem suas próprias decisões e melhorar seu estilo de vida ${ }^{(10)}$.

Este estudo teve limitações, a inclusão de um pequeno tamanho da amostra, e recomenda-se que o uso de jogos seja avaliado por um maior número de utilizadores, assim como a falta de um grupo de comparação. Outra limitação foi o facto de não ter sido avaliado pelos utentes o que permitiria uma avaliação mais alargada, propondo-se esta abordagem para estudos futuros. Por outro lado, faltam evidências empíricas do impacto nos utilizadores, estudantes e utentes, quanto aos resultados das estratégias de aprendizagem ativa. Mais estudos são sugeridos para analisar como as estratégias de aprendizado ativo, como os jogos, afetam os resultados da aprendizagem, incluindo os resultados da melhoria da saúde das organizações.

\section{CONCLUSÃO}

O jogo foi uma estratégia de ensino útil que os estudantes avaliaram positivamente. Acredita-se que o jogo Reabilit' Arte é uma estratégia de educação para a saúde em que os utilizadores se implicam e são implicados em seu processo de pensar, de elaborar novos sentidos, de conhecer e de agir, favorecendo o empoderamento e uma transição saúde/doença eficaz. 0 jogo foi bem-sucedido ao permitir que um grande volume de informações fosse coberto por uma atividade, contribuindo para um maior envolvimento do utilizador, numa aprendizagem ativa ao realizar as tarefas exigidas no interagir com o jogo educativo. Dado o aumento do número de artroplastias da anca e a diminuição dos tempos de internamento, e se o caminho é facilitar a recuperação pós-operatória, os utentes devem ter instruções claras sobre como progredir na sua reabilitação de forma independente.

0 presente estudo foi realizado num contexto de prática hospitalar, integrando estudantes da póslicenciatura em enfermagem de reabilitação no ensino clínico da pessoa com afeções orto traumatológicas e conjuntivas, podendo ser replicado e testado em outros contextos similares, designadamente na comunidade. Os resultados evidenciam uma boa aceitação do jogo como estratégia de educação para a saúde que necessita ser incorporada. De futuro sugere-se mais estudos de investigação nesta área que possam corroborar os dados apresentados e que possam valorizar o trabalho e o impacto que as intervenções do enfermeiro especialistas em enfermagem de reabilitação têm na qualidade de vida dos utentes. 


\section{REFERÊNCIAS BIBLIOGRÁFICAS}

1. Schmid J, Chênes $C$, Chagué $S$, Hoffmeyer $P$, Christofilopoulos $P$, Bernardoni $M$, et al. MyHip: supporting planning and surgical guidance for a better total hip arthroplasty: A pilot study. Int $J$ Comput Assist Radiol Surg. 2015;10(10):1547-56. Doi: 10.1007/s11548-015-1177-2

2. Kennedy D, Wainwright A, Pereira L, Robarts S, Dickson P, Christian J, et al. A qualitative study of patient education needs for hip and knee replacement. BMC Musculoskelet Disord. 2017;18(1):413. Doi:10.1186/s12891-017-1769-9

3. Portugal. Ministério da Saúde. Regulamento n. ${ }^{\circ} 392 / 2019$, de 03 de maio de 2019. Regulamento das Competências Específicas do Enfermeiro Especialista em Enfermagem de Reabilitação. Diário da República. 2019; 2. ${ }^{\text {a }}$ série. p 13565-13568. Disponível em: https://dre.pt/web/guest/pesquisa/-

/search/122216893/details/normal?l=1

4. Wainwright TW, Burgess LC. To what extent do current total hip and knee replacement patient information resources adhere to enhanced recovery after surgery principles? Physiother. 2018 ;104(3):327-37. Doi: 10.1016/j.physio.2018.05.002

5. Wang X, Hunter DJ, Vesentini G, Pozzobon D, Ferreira ML. Technology-assisted rehabilitation following total knee or hip replacement for people with osteoarthritis: a systematic review and meta-analysis. BMC Musculoskelet Disord. 2019;20(1):506. Doi:10.1186/s12891-019-2900-x

6. Rosner BI, Gottlieb M, Anderson WN. Effectiveness of an Automated Digital Remote Guidance and Telemonitoring Platform on Costs, Readmissions, and Complications After Hip and Knee Arthroplasties. J Arthroplasty. 2018;33(4):988. Doi: 10.1016/j.arth.2017.11.036

7. Dayucos A, French LA, Kelemen A, Liang Y, Sik Lanyi C. Creation and Evaluation of a Preoperative Education Website for Hip and Knee Replacement Patients-A Pilot Study. Med. 2019;55(2). Doi: 10.3390/medicina55020032

8. Ling Y, Ter Meer LP, Yumak Z, Veltkamp RC. Usability Test of Exercise Games Designed for Rehabilitation of Elderly Patients After Hip Replacement Surgery: Pilot Study. JMIR serious games 2017; 5(4):e19. Doi: 10.2196/games.7969

9. D'Aprile G, Ligorio MB, Ulloa Severino A. How Serious Games for Health Enhance Empowerment-Related Patient Education: The Impact of Gender. Technology, Knowledge and Learning. 2019;24(3):325-40. Doi:10.1007/s10758-017-9344-x

10. Sharifzadeh N, Kharrazi H, Nazari E, Tabesh H, Edalati Khodabandeh M, Heidari S, et al. Health Education Serious Games Targeting Health Care Providers, Patients, and Public Health Users: Scoping Review. JMIR serious games. 2020;8(1):e13459. Doi: 10.2196/13459

11. Jansson $M$, Koivisto J, Pikkarainen $M$. Identified opportunities for gamification in the elective primary fast-track total hip and knee arthroplasty journey: Secondary analysis of healthcare professionals' interviews. J Clin Nurs. 2020;29(13-14):2338-51.
Doi:10.1111/jocn.15246

12. Byra J, Czernicki K. The Effectiveness of Virtual Reality Rehabilitation in Patients with Knee and Hip Osteoarthritis. J Clin Med. 2020;9(8). Doi:10.3390/jcm9082639

13. Fernandes Carla Sílvia, Ângelo Margareth. Estratégias lúdicas utilizadas em enfermagem - Uma revisão integrativa. av.enferm. 2018 Abr; 36( 1 ): 88-98. Doi: 10.15446/av.enferm.v36n1.63553

14. Cruz JG. Vivendo o jogo ou jogando a vida? Notas sobre jogos (digitais) e educação em meio à cultura ludificada. Rev. Bras. Ciênc. Esporte. 2017; 39( 3 ): 226-232. Doi: 10.1016/j.rbce.2017.02.012

15. Fernandes CS, Magalhaes B, Santos C, Martines-Galiano J. The Use of Games in the Self-Management of Oncological Disease: An Integrative Literature Review. Online J Nurs Inform. 2019. 23(3):7-1.

16. Naderifar, Mahin \& Goli, Hamideh \& Ghaljaei, Fereshteh. Snowball Sampling: A Purposeful Method of Sampling in Qualitative Research. Strides in Development of Medical Education. 2017. Doi:10.5812/sdme.67670

17. Fernandes, C S; Martins, M; Gomes, B. Construir um instrumento de avaliação de estratégia de formação (IAEF). Boletim Cientifico da Escola Superior de Enfermagem de Santa Maria, 2014. 6:p. 11 $-13$

18. Bardin L. Análise de Conteúdo. Lisboa: Edições; 2009.

19. Boctor L. Active-learning strategies: the use of a game to reinforce learning in nursing education: a case study. Nurse Educ Pract 2013 Mar.; 13(2): 96-100. Doi: 10.1016/j.nepr.2012.07.010

20. Graafland M, Dankbaar M, Mert A, Lagro J, De Wit-Zuurendonk L, Schuit S, Schaafstal A, Schijven M. How to Systematically Assess Serious Games Applied to Health Care JMIR Serious Games 2014;2(2):e11. Doi: 10.2196/games.3825

21. Boyle EA, Hainey T, Connolly TM, Gray G, Earp J, Ott M, et al. An update to the systematic literature review of empirical evidence of the impacts and outcomes of computer games and serious games. Comput Educ [Internet]. 2016 Mar [cited 2020 Mar 28];94:178-92. Doi: 10.1016/j.compedu.2015.11.003

22. Wang $X$, Hunter DJ, Vesentini G, Pozzobon D, Ferreira ML. Technology-assisted rehabilitation following total knee or hip replacement for people with osteoarthritis: a systematic review and meta-analysis. BMC Musculoskelet Disord.. 2019;20(1):506. Doi:10.1186/s12891-019-2900-x

23. Zavala J, Fitace F, León M, Ponce F, Gutiérrez H. Resultados funcionales tras entrenamiento fisioterapéutico que incluye la realidad virtual en mayores de 60 años con artroplastia total de cadera: estudio descriptivo. Fisioter. 2017;39(6):236-41.

24. Abujaber S, Gillispie G, Marmon A, Zeni J Jr. Validity of the Nintendo Wii Balance Board to assess weight bearing asymmetry during sit-to-stand and return-to-sit task. Gait Posture. 2015;41(2):676-82. Doi:10.1016/j.gaitpost.2015.01.023 